\title{
Quantitative and qualitative assessment of plasma cell dyscrasias in dual-layer spectral CT
}

\author{
S. C. Brandelik ${ }^{1}$ - S. Skornitzke ${ }^{1} \cdot$ T. Mokry ${ }^{1} \cdot$ S. Sauer ${ }^{2} \cdot$ W. Stiller ${ }^{1} \cdot$ J. Nattenmüller ${ }^{1} \cdot$ H. U. Kauczor ${ }^{1} \cdot$ T. F. Weber ${ }^{1}$. \\ T. D. Do ${ }^{1}$
}

Received: 23 December 2020 / Accepted: 19 February 2021 / Published online: 30 March 2021

(C) The Author(s) 2021

\begin{abstract}
Objectives Virtual non-calcium (VNCa) images could improve assessment of plasma cell dyscrasias by enhancing visibility of bone marrow. Thus, VNCa images from dual-layer spectral CT (DLCT) were evaluated at different calcium suppression (CaSupp) indices, correlating results with apparent diffusion coefficient (ADC) values from MRI.

Methods Thirty-two patients with initial clinical diagnosis of a plasma cell dyscrasia before any chemotherapeutic treatment, who had undergone whole-body low-dose DLCT and MRI within 2 months, were retrospectively enrolled. VNCa images with CaSupp indices ranging from 25 to 95 in steps of 10, conventional CT images, and ADC maps were quantitatively analyzed using region-of-interests in the vertebral bodies C7, T12, L1-L5, and the iliac bone. Independent two-sample $t$-test, Wilcoxon-signedrank test, Pearson's correlation, and ROC analysis were performed.

Results Eighteen patients had a non-diffuse, 14 a diffuse infiltration in conventional MRI. A significant difference between diffuse and non-diffuse infiltration was shown for VNCa-CT with CaSupp indices from 55 to 95 , for conventional CT, and for ADC (each $p<0.0001$ ). Significant quantitative correlation between VNCa-CT and MRI could be found with strongest correlation at CaSupp index 65 for L3 $(r=0.68, p<0.0001)$ and averaged L1-L5 $(r=0.66, p<0.0001)$. The optimum CT number cut-off point for differentiation between diffuse and non-diffuse infiltration at CaSupp index 65 for averaged L1-L5 was $-1.6 \mathrm{HU}$ (sensitivity $78.6 \%$, specificity $75.0 \%$ ).

Conclusion Measurements in VNCa-CT showed the highest correlation with ADC at CaSupp index 65. VNCa technique may prove useful for evaluation of bone marrow infiltration if MRI is not feasible.

Key Points

- VNCa-CT images can support the evaluation of bone marrow infiltration in plasma cell dyscrasias.

- VNCa measurements of vertebral bodies show significant correlation with ADC in MRI.

- Averaging L1-L5 at CaSupp index 65 allowed quantitative detection of infiltration comparable to MRI ADC.
\end{abstract}

Keywords Computed tomography $\cdot$ Multiple myeloma $\cdot$ Plasma cell dyscrasia $\cdot$ Virtual non-calcium $\cdot$ Dual-layer spectral CT

$\overline{\text { Simone Brandelik and Stephan Skornitzke have contributed equally to }}$ this work.

T. D. Do

thuy.do@med.uni-heidelberg.de

1 Clinic of Diagnostic and Interventional Radiology (DIR), Heidelberg University Hospital, Im Neuenheimer Feld 420, 69120 Heidelberg, Germany

2 Medical Department V, Hematology/Oncology/Rheumatology, Heidelberg University Hospital, Im Neuenheimer Feld 410, 69120 Heidelberg, Germany

$\begin{array}{ll}\text { Abbreviations } & \\ \text { AU } & \text { Arbitrary unit } \\ \text { CaSupp index } & \text { Calcium suppression index } \\ \text { CNR } & \text { Contrast-to-noise ratio } \\ \text { DLCT } & \text { Dual-layer spectral detector CT } \\ \text { FOV } & \text { Field of view } \\ \text { MGUS } & \begin{array}{l}\text { Monoclonal gammopathy of undetermined } \\ \text { significance }\end{array} \\ \text { SD } & \text { Standard deviation } \\ \text { SMM } & \text { Smoldering multiple myeloma } \\ \text { VNCa } & \text { Virtual non-calcium }\end{array}$




\section{Introduction}

Multiple myeloma (MM) and its precursors monoclonal gammopathy of undetermined significance (MGUS) and smoldering myeloma (SMM) are characterized by monoclonal proliferation of plasma cells with the spine as the predominant site of active hematopoietic bone marrow being the primary target. For MM, different infiltration patterns of the bone marrow have been described in conventional MRI [1] —normal, focal, diffuse, mixed, and variegated salt-and-pepper pattern-and were shown to have a prognostic impact $[2,3]$. Diffusion-weighted imaging sequences (DWIs) and the apparent diffusion coefficient (ADC) in MRI have been shown to correlate with cellularity of the bone marrow in MM [4, 5]. ADC values differ significantly between infiltration patterns [6]. The International Myeloma Working Group currently recommends whole-body low-dose CT as the imaging modality of choice for the initial assessment in monoclonal plasma cell diseases based on the importance of detection of osteolytic lesions for disease definition and detection of imminent disabling fractures [7, 8]. Only in certain cases, such as equivocal CT results in MGUS and inconclusive CT in suspected SMM and MM, MRI is currently recommended as a further imaging modality [7]. However, particularly diffuse hypercellularity is obscured on conventional $\mathrm{CT}$, and to date, MRI is the best non-invasive modality to detect bone marrow infiltration which is not necessarily associated with osteolytic bone destruction $[7,9]$.

In dual-layer spectral CT (DLCT), two different detector layers atop each other absorb different parts of the polychromatic patient-attenuated X-ray spectrum instead of activating a second X-ray tube or rapid-voltage switching tube before performing the examination. The benefit is the opportunity of retrospective spectral analyses and the selective depiction or suppression of materials, e.g., uric acid [10], iodine [11], or calcium [12]. In virtual non-calcium (VNCa) images, the osseous component is removed from the spectral base data in order to improve visualization of bone marrow. The degree of calcium suppression depends on the calcium suppression (CaSupp) index, which defines the calcium composition level.

Using the dual-source dual-energy technique, significantly increased $\mathrm{VNCa}-\mathrm{CT}$ numbers in MRI-confirmed bone marrow lesions compared to non-infiltrated bone marrow were found [13] and VNCa-CT numbers differed significantly between different infiltration patterns [14]. The aim of this study is to correlate conventional and $\mathrm{VNCa}-\mathrm{CT}$ images with MRI images to evaluate the quantitative and qualitative assessment of bone marrow infiltration in DLCT compared to the gold standard MRI.

\section{Material and methods}

\section{Ethics approval and consent}

This retrospective exploratory single-center study was approved by the local review board. The need for written informed consent was waived.

\section{Clinical data selection and study design}

Patients were screened in the hospital and radiological information system (I.S.-H.*med., SAP; Centricity RIS-i, GE Healthcare) for study inclusion. Patients with an initial diagnosis of a plasma cell dyscrasia (MM, SMM or MGUS) and routinely performed whole-body low-dose DLCT and wholebody MRI within 2 months of each other from August 2018 until October 2019 were enrolled into the study. Exclusion criteria were prior anti-myeloma chemotherapeutic treatment, additional solid malignancies, extensive imaging artifacts, or missing spectral data.

The patient cohort consisted of 38 patients matching the inclusion criteria, of whom 6 patients were excluded due to missing spectral information. A diffuse infiltration of the axial bone marrow was present in 14 cases and a non-diffuse infiltration in 18 cases. Twenty-seven patients were diagnosed with MM (14 with diffuse infiltration and 13 with non-diffuse infiltration), 4 patients with SMM (all with non-diffuse infiltration), and 1 patient with MGUS (non-diffuse infiltration) (Table 1). Median age at time of CT was 62.5 years (range 38-78). Median time between CT and MRI was 2.5 days (range 0-50).

\section{DLCT acquisition and post-processing}

Non-contrast $\mathrm{CT}$ acquisitions were performed as helical scans from the vertex of the skull to the knees using a dual-layer detector technique (IQon Spectral CT, Philips) with the

Table 1 Study population with type of plasma cell dyscrasia and infiltration

\begin{tabular}{ll}
\hline Plasma cell dyscrasia type & Number of patients \\
\hline MGUS & 1 \\
MGUS diffuse infiltration & 0 \\
MGUS non-diffuse infiltration & 1 \\
Smoldering myeloma & 4 \\
Smoldering myeloma diffuse infiltration & 0 \\
Smoldering myeloma non-diffuse infiltration & 4 \\
Multiple myeloma & 27 \\
Multiple myeloma diffuse infiltration & 14 \\
Multiple myeloma non-diffuse infiltration & 13 \\
\hline
\end{tabular}


following acquisition parameters: tube voltage $120 \mathrm{kV}_{\mathrm{p}}$, dose right index 15 (automated, attenuation-based dose modulation), average tube current-time product $93 \mathrm{mAs}$, mean computed tomography dose index $\left(\mathrm{CTDI}_{\mathrm{vol}}\right) 6.5 \mathrm{mGy}$ (standard deviation [SD] $0.7 \mathrm{mGy}$, range 5.1-7.6 $\mathrm{mGy}, 32 \mathrm{~cm}$ bodyphantom), pitch 1.0, gantry rotation time of $0.75 \mathrm{~s}$, and collimation $64 \times 0.625 \mathrm{~mm}$. The field of view (FOV) was variable depending on patient body volume.

VNCa image reconstructions of DLCT data and CT number measurements were performed with the manufacturer's dedicated image post-processing software (IntelliSpace Portal Version 11, Philips). VNCa images were reconstructed with CaSupp indices ranging from 25 to 95 in steps of 10 with minimum visibility of bony structures at index 25 and maximum visibility at index 95 . Reconstruction was performed with slice thickness $3 \mathrm{~mm}$, increment $1.5 \mathrm{~mm}$, sharp kernel YA, and iterative reconstruction with iDose level 2 for conventional images and kernel $\mathrm{B}$ for $\mathrm{VNCa}$ images.

Using the same software, regions of interest (ROIs) were positioned manually in the vertebral bodies C7, Th12, L1-L5, and the right iliac bone to measure the respective $\mathrm{CT}$ numbers (mean value and SD). ROI sizes were $50 \mathrm{~mm}^{2}$ in $\mathrm{C} 7$ and 150 $\mathrm{mm}^{2}$ in all other locations $\left( \pm 5 \mathrm{~mm}^{2}\right)$. Inclusion of focal lesions into the ROI was avoided, if visible on CT or MRI. Pretreated vertebrae-radiotherapy, kyphoplasty, or spondylodesis - and adjacent vertebrae with treatmentassociated artifacts or with unavoidable measurement of sclerosis were excluded. To ensure comparability, ROIs were copied between different CaSupp indices and conventional images.

A contrast-to-noise ratio (CNR) was calculated for each measurement location and for each CaSupp index:

$\mathrm{CNR}=\frac{\left|\mu_{\text {diffuse }}-\mu_{\text {non-diffuse }}\right|}{\sigma}$

Here, $\mu_{\text {non }- \text { diffuse }}$ is the mean CT number of all patients with non-diffuse infiltration, $\mu_{\text {diffuse }}$ is the mean CT number of all patients with diffuse infiltration, and $\sigma$ is the mean of all measured SDs for the measurement location and the CaSupp index.

\section{MRI acquisition and analysis}

Whole-body MRI was performed on a 1.5 Tesla MRI (MAGNETOM AvantoFit, Siemens Healthineers) with the following imaging protocol: unenhanced coronal T1weighted turbo spin echo sequences, short tau inversion recovery (STIR) sequences, and transversal DWI sequences ( $b$-values 50 and $800 \mathrm{~s} / \mathrm{mm}^{2}$, five blocks of $46 \times$ $46 \mathrm{~cm}$ ) with calculation of ADC maps. A signal loss in T1 and concurrent signal increase in STIR was defined as plasma cell infiltration of the bone marrow-either with signal alteration of focal areas within a normal fat signal or with diffuse homogeneous signal alteration of the spine [1]. By means of a consensus read by all radiologists, the infiltration pattern of each patient was considered either as diffuse (defined as diffuse infiltration, variegated salt-andpepper pattern [1] or intense disseminated focal infiltration) or as non-diffuse (defined as normal pattern or sporadic focal infiltration). T1 and STIR sequences were also screened to avoid inclusion of large focal lesions into the quantitative evaluation. The consensus read was considered standard of reference for this study.

In the institutional PACS (GE Healthcare), ROIs were positioned manually in the ADC maps of the vertebral bodies C7, Th12, L1-L5, and the right iliac bone to obtain the respective mean signal intensity (ADC value) and SD. ROI sizes were $50 \mathrm{~mm}^{2}$ in $\mathrm{C} 7,200-250 \mathrm{~mm}^{2}$ in Th12, L1-L5, and $100-150 \mathrm{~mm}^{2}$ in the iliac bone $\left( \pm 5 \mathrm{~mm}^{2}\right)$. Slice thickness was $5 \mathrm{~mm}$. Inclusion of focal lesions was avoided if determinable. CNR for each measurement location and CaSupp index was calculated for ADC values as described above for $\mathrm{CT}$ numbers.

\section{Image analysis}

Quantitative and qualitative image analysis was performed independently by two radiologists ( 4 and 8 years of experience).

For qualitative assessment of $\mathrm{VnCa}$ images, areas within the bone marrow that visually showed a density comparable to that of the erector spinae or gluteal muscles were defined as areas with plasma cell infiltration. Using the same criteria as described above for MRI, a decision was made for non-diffuse (Fig. 1) or diffuse infiltration (Fig. 2) on VNCa images at CaSupp index 65, which showed the strongest correlation to $\mathrm{ADC}$ in the quantitative evaluation.

\section{Statistical analysis}

Statistical analysis was performed using SAS Version 9.4 (SAS Institute Inc.). Descriptive statistics were calculated, determining means and SDs for normal distributed data. For assessment of significant differences in CT numbers between the infiltration patterns, an independent twosample $t$-test was applied (conventional and VNCa-CT) following a normal distribution. For MRI, a Wilcoxonsigned-rank test was applied for the same question (nonnormal distribution of data). Correlations between VNCa$\mathrm{CT}$ numbers and ADC values were calculated using Pearson's correlation. Receiver operating characteristic (ROC) analysis with calculation of the area under the ROC curve (AUC) and Youden's J statistic were performed to determine the cut-off for the mean $\mathrm{VNCa}-\mathrm{CT}$ 


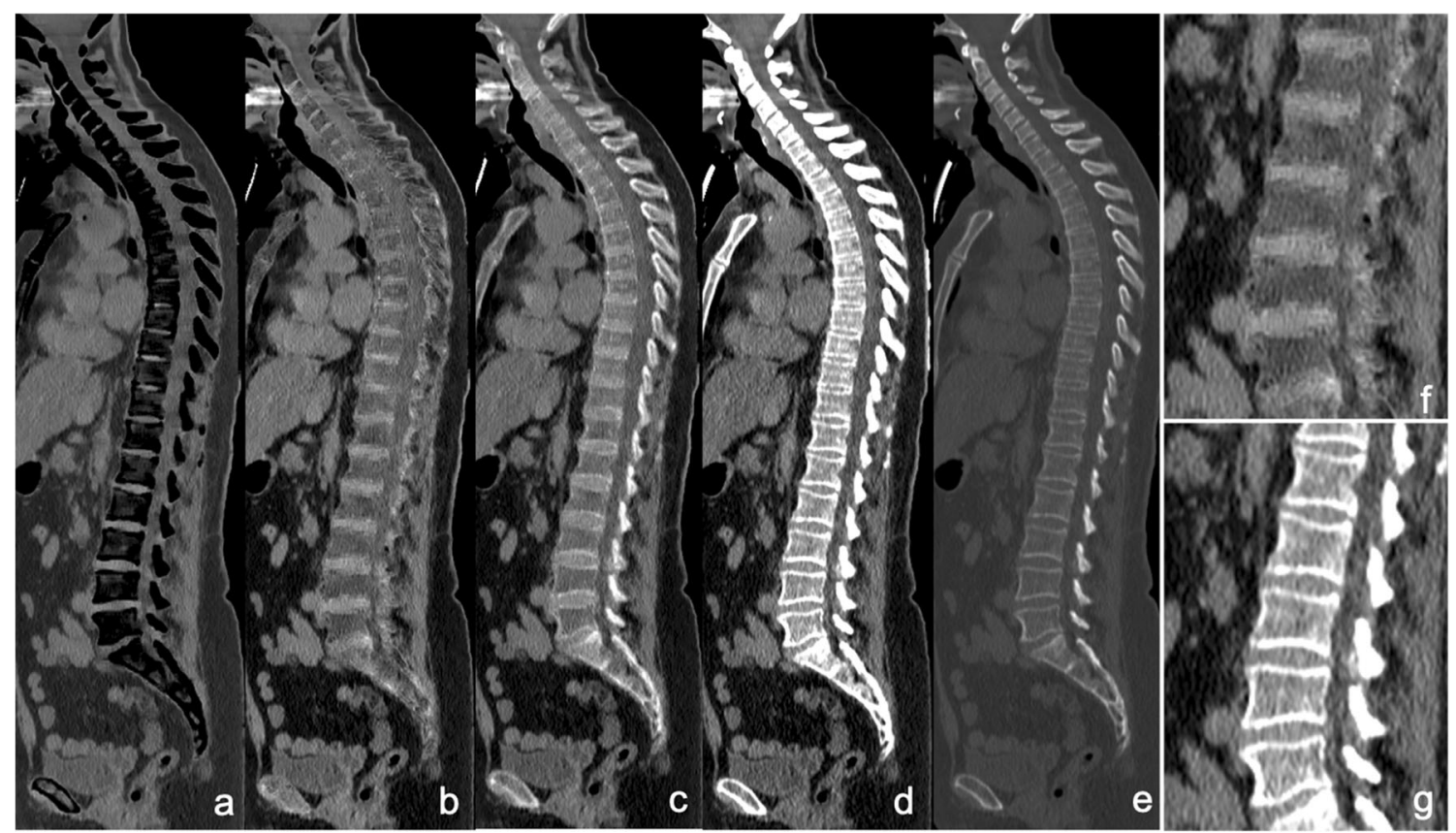

Fig. 1 Patient with non-diffuse infiltration. a CaSupp index 25; b CaSupp index 65; c CaSupp index 95; d conventional CT soft tissue window; e conventional CT bone window; $\mathbf{f}, \mathbf{g}$ magnification of $\mathbf{b}$ and $\mathbf{d}$ for L1-5

number of L1-L5 and of L3 at CaSupp index 65, which showed the highest correlation to ADC. AUCs were compared with each other using a large-sample $\chi^{2}$ test. The intraclass correlation coefficient (ICC) was calculated for determination of the interrater reliability of the quantitative and qualitative assessment. The significance level for statistical testing was set at $p<0.05$.

\section{Results}

\section{Quantitative analysis}

Table 2 shows CT numbers and ADC values of different vertebral bodies, infiltration types, and CaSupp indices. CT numbers in $\mathrm{VNCa}$ images were significantly different between

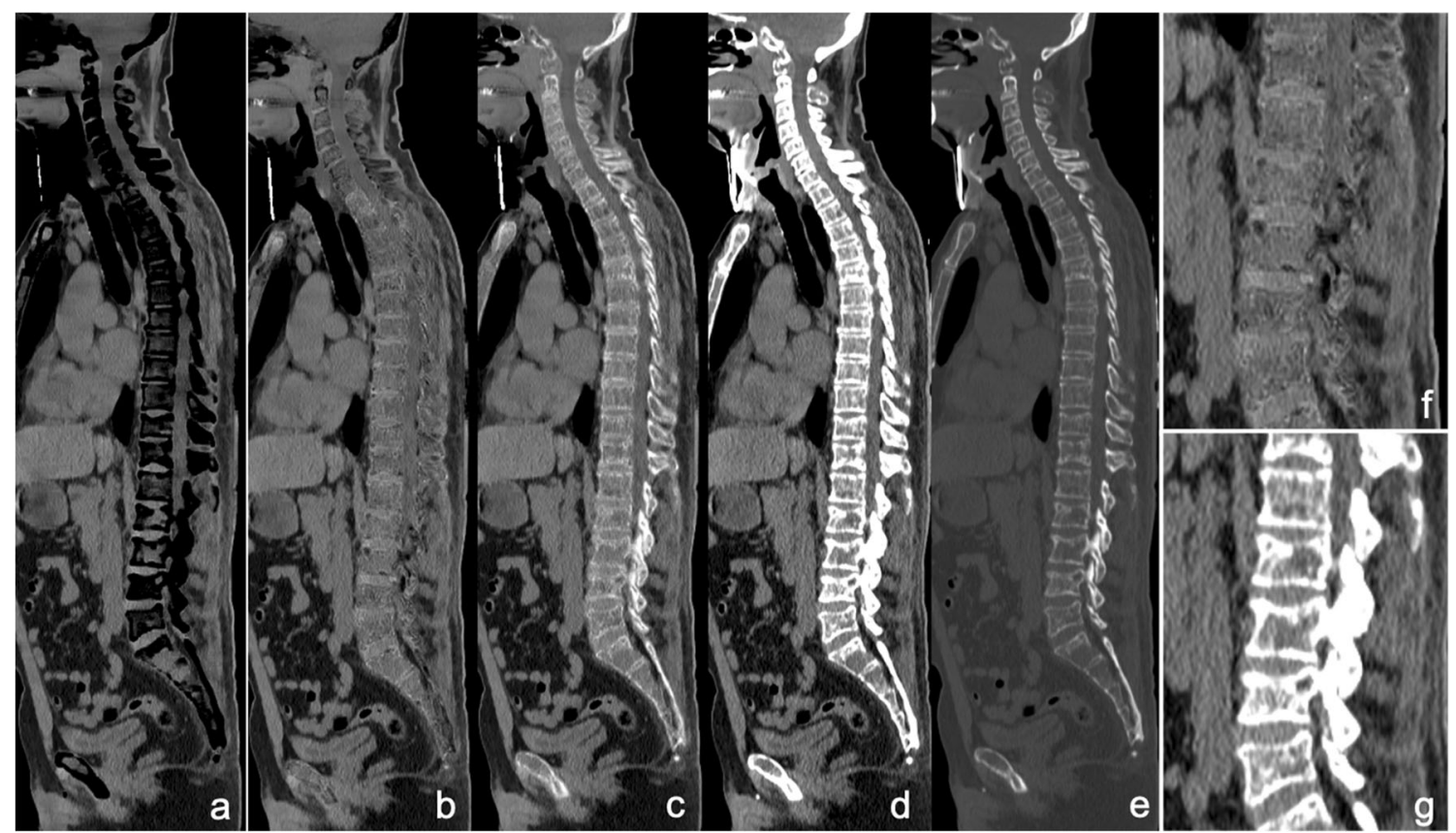

Fig. 2 Patient with diffuse infiltration; a CaSupp index 25; b CaSupp index 65; c CaSupp index 95; $\mathbf{d}$ conventional CT soft tissue kernel; e conventional CT sharp kernel; $\mathbf{f}, \mathbf{g}$ magnification of $\mathbf{b}$ and $\mathbf{d}$ for L1-5 


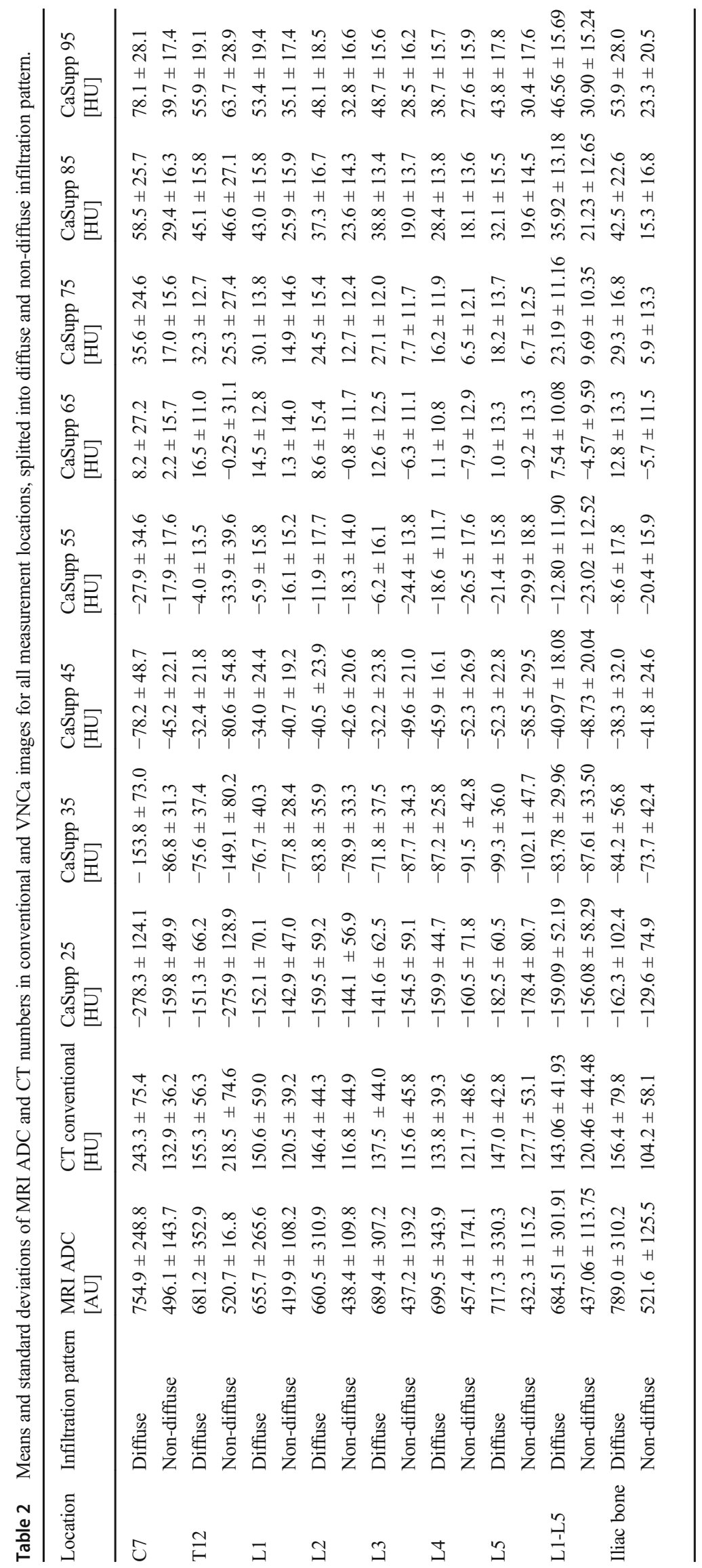


diffuse and non-diffuse infiltration at CaSupp indices from 55 to 95 (each with $p<0.0001$ ). CT numbers in conventional images as well as ADC values also showed significant differences between both groups (both $p<0.0001$ ).

Figure 3 summarizes the statistical results regarding the correlation between $\mathrm{CT}$ numbers and ADC values for different CaSupp indices and measurement locations. Regardless of the location, the highest correlation of VNCa-CT numbers and ADC values was at CaSupp indices from 55 to 95 (Pearson's $r$ between 0.26 and 0.68 ; all $p$ $<0.05$ ). Correlation of CT numbers in conventional images and ADC values was lower than that of CT numbers measured at CaSupp indices from 55 to 95 (Pearson's $r$ between 0.05 and 0.23 ; all $p>0.05$ ). No significant correlation of CT numbers and ADC values could be shown in the vertebra $\mathrm{C} 7$ for all CaSupp indices. For each CaSupp index, the highest correlation of CT numbers and ADC values was in the vertebra L3. The strongest correlation of $\mathrm{VNCa}-\mathrm{CT}$ numbers and ADC values for averaged L15 vertebrae $(r=0.66 ; p<0.0001)$ and L3 vertebra $(r=$ $0.68 ; p<0.0001$ ) was found at CaSupp index 65 .

ROC analysis for the differentiation between diffuse and non-diffuse infiltration for mean values of L1-5 and L3 alone revealed similar AUCs for DLCT at CaSupp index $65(\mathrm{AUC}=0.819$ and $\mathrm{AUC}=0.885)$ (Fig. 4a) and for ADC $(\mathrm{AUC}=0.863$ and $\mathrm{AUC}=0.846)($ Fig. $4 \mathrm{~b})$, but lower AUCs for conventional $\mathrm{CT}(\mathrm{AUC}=0.641$ and $\mathrm{AUC}=$ 0.636) (Fig. 4c). For mean values of L1-5, the AUC for conventional CT was significantly below that for $\mathrm{VNCa}-$ CT with CaSupp index $65(p=0.048)$ and $\operatorname{ADC}(p=0.01)$, while there was no significant difference in AUC between VNCa-CT with CaSupp index 65 and ADC $(p=0.526)$. The optimum cut-off point for VNCa-CT in L1-L5 was $-1.6 \mathrm{HU}$, with higher values indicating a diffuse infiltration with a sensitivity of $78.6 \%$ and a specificity of $75.0 \%$ (Fig. 5). The cut-off for ADC was 487.6 AU with values above indicating a diffuse infiltration with a sensitivity of $78.6 \%$ and a specificity of $86.1 \%$. Considering L3 alone, the optimum cut-off points were $3.1 \mathrm{HU}$ for VNCa-CT (sensitivity: $78.6 \%$, specificity $88.9 \%$ ) and $516.7 \mathrm{AU}$ for ADC (sensitivity: $75.0 \%$, specificity $86.1 \%$ ).

On average, CNR of VNCa images was highest in L3. For VNCa images, the CNR for CaSupp indices from 65 to 95 exceeded the CNR for conventional CT on average. For CaSupp indices from 65 to 95 , the highest CNR was found in the iliac bone.

Table 3 summarizes the CNR for different locations and image data in conventional CT, VNCa images, and MRI.

\section{Qualitative analysis}

At CaSupp index 65, diffuse infiltration was detected with a sensitivity and specificity of $83.3 \%$ and $78.6 \%$ for reader 1 and $92.3 \%$ and $68.4 \%$ for reader 2 . Accordingly, average sensitivity and specificity were $87.8 \%$ and $73.5 \%$ for qualitative analysis.

\section{Interrater reliability of quantitative and qualitative analysis}

The interrater reliability of all quantitative measurements in CT was very high with an ICC of 0.98 .

The interrater reliability of the qualitative assessment for the differentiation between diffuse versus non-diffuse infiltration in DLCT at CaSupp index 65 was substantial with an ICC of 0.69 [15].

\begin{tabular}{|c|c|c|c|c|c|c|c|c|c|c|c|c|c|c|c|c|c|c|}
\hline & \multicolumn{2}{|l|}{ T12 } & \multicolumn{2}{|l|}{ C7 } & \multicolumn{2}{|l|}{ L1 } & \multicolumn{2}{|l|}{ L2 } & \multicolumn{2}{|l|}{ L3 } & \multicolumn{2}{|l|}{ L4 } & \multicolumn{2}{|l|}{ L5 } & \multicolumn{2}{|l|}{ L1-L5 } & \multicolumn{2}{|l|}{ RIB } \\
\hline & $r$ & $p$ & $r$ & $p$ & $r$ & $p$ & $r$ & $p$ & $r$ & $p$ & $r$ & $p$ & $r$ & $p$ & $r$ & $p$ & $r$ & $p$ \\
\hline $\begin{array}{c}\text { CaSupp } \\
\text { Index } 95 \\
\end{array}$ & 0.26 & 0.0367 & 0.06 & 0.6399 & 0.31 & 0.0133 & 0.34 & 0.0056 & 0.48 & $<.0001$ & 0.5 & 0.0002 & 0.4 & 0.001 & 0.44 & 0.0003 & 0.39 & 0.0013 \\
\hline $\begin{array}{c}\text { CaSupp } \\
\text { Index } 85\end{array}$ & 0.31 & 0.0118 & 0.02 & 0.897 & 0.35 & 0.0045 & 0.37 & 0.0024 & 0.56 & $<.0001$ & 0.5 & $<<0001$ & 0.47 & $<.0001$ & 0.51 & $<.0001$ & 0.42 & 0.0005 \\
\hline $\begin{array}{c}\text { CaSupp } \\
\text { Index } 75\end{array}$ & 0.37 & 0.0023 & $\mid-0.03$ & 0.8212 & 0.36 & 0.0033 & 0.4 & 0.001 & 0.64 & $<.0001$ & 0.6 & $<<.0001$ & 0.55 & $<.0001$ & 0.60 & $<.0001$ & 0.46 & 0.0001 \\
\hline $\begin{array}{c}\text { CaSupp } \\
\text { Index } 65\end{array}$ & 0.43 & 0.0004 & $\mid-0.07$ & 0.5827 & 0.37 & 0.0027 & 0.4 & 0.0011 & 0.68 & $<.0001$ & 0.6 & $<<0001$ & 0.56 & $<.0001$ & 0.66 & $<.0001$ & 0.46 & 0.0001 \\
\hline $\begin{array}{c}\text { CaSupp } \\
\text { Index } 55 \\
\end{array}$ & 0.43 & 0.0004 & $\mid-0.11$ & 0.4169 & 0.31 & 0.0122 & 0.33 & 0.008 & 0.63 & $<.0001$ & 0.5 & $<<.0001$ & 0.45 & 0.0002 & 0.58 & $<.0001$ & 0.28 & 0.0227 \\
\hline $\begin{array}{c}\text { CaSupp } \\
\text { Index } 45\end{array}$ & 0.37 & 0.0023 & $\mid-0.13$ & 0.3345 & 0.21 & 0.0969 & 0.21 & 0.0939 & 0.49 & $<.0001$ & 0.4 & 0.0013 & 0.31 & 0.0116 & 0.40 & 0.0011 & 0.07 & 0.5623 \\
\hline $\begin{array}{c}\text { CaSupp } \\
\text { Index } 35\end{array}$ & 0.3 & 0.0179 & $\mid-0.13$ & 0.3424 & 0.11 & 0.3877 & 0.1 & 0.4249 & 0.36 & 0.0037 & 0.3 & $\mid 0.0321$ & 0.21 & 0.1012 & 0.25 & 0.0489 & $\mid-0.05$ & 0.6969 \\
\hline $\begin{array}{c}\text { CaSupp } \\
\text { Index } 25 \\
\end{array}$ & 0.23 & 0.0711 & -0.09 & 0.4885 & 0.04 & 0.7511 & 0.04 & 0.7649 & 0.25 & 0.0478 & 0.2 & 0.1575 & 0.13 & 0.3049 & 0.15 & 0.2500 & $\mid-0.11$ & 0.3736 \\
\hline $\begin{array}{c}\mathrm{CT} \\
\text { conventional }\end{array}$ & 0.05 & 0.6967 & 0.19 & 0.1564 & 0.16 & 0.2076 & 0.17 & 0.1726 & 0.12 & 0.3395 & 0.1 & 0.3405 & 0.16 & 0.2069 & 0.16 & 0.2017 & 0.23 & 0.0627 \\
\hline
\end{tabular}

Fig. 3 Heat map of Pearson's correlation $r$ and $p$ value between CT numbers and ADC values for different CaSupp indices and measurement locations 

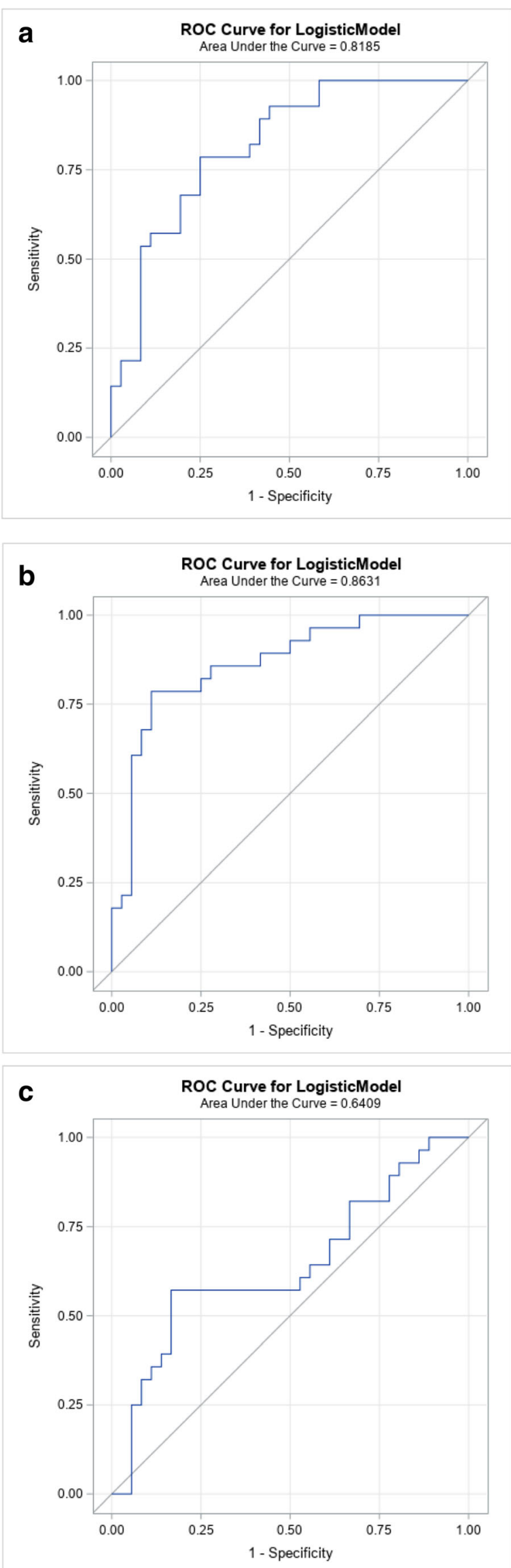

Fig. 4 ROC curves for CT and MRI; a ROC curve analysis: good differentiation between diffuse and non-diffuse infiltration for mean L15 vertebrae at CaSupp index 65 with AUC of 0.8185 ; $\mathbf{b}$ ROC curve of the MRI ADC for mean of L1-5 vertebrae with AUC of 0.8631. $\mathbf{c}$ ROC curve of conventional CT for mean of L1-5 vertebrae with AUC of 0.6409

\section{Discussion}

VnCa imaging on DLCT providing spectral data gives the opportunity to detect osteolytic bone lesions and evaluate bone marrow involvement in a quantitative and qualitative manner [16]. Therefore, our study aimed for a detailed quantitative assessment of the performance of VNCa-DLCT in the evaluation of bone marrow infiltration in plasma cell dyscrasias compared to MRI, considering CaSupp index and location of measurement.

The results show that differentiating MRI-confirmed diffuse infiltration from non-diffuse infiltration in quantitative DLCT is possible at high CaSupp indices, also showing comparable ROC curves and AUCs to ADC measurements for VNCa-CT. Strong correlations to ADC suggest that bone marrow infiltration can best be assessed in $\mathrm{VNCa}$ images at CaSupp index 65. The threshold of $-1.6 \mathrm{HU}$ for the average from measurements in L1-L5 vertebrae can be used for differentiation between diffuse and non-diffuse infiltration, reaching a sensitivity of $78.6 \%$ and a specificity of $75.0 \%$.

The highest correlation of CT and MRI in the L3 vertebra might be due to the fact that L3 has the largest distance to junctions between kyphosis and lordosis with less likely sclerosis/fractures. The poor results for $\mathrm{C} 7 \mathrm{can}$ be attributed to beam hardening artifacts in CT-which might be overcome with future photon-counting detector technique [17] - and to position-provoked oblique sectioning of the small $\mathrm{C} 7$ in transversal MRI slices.

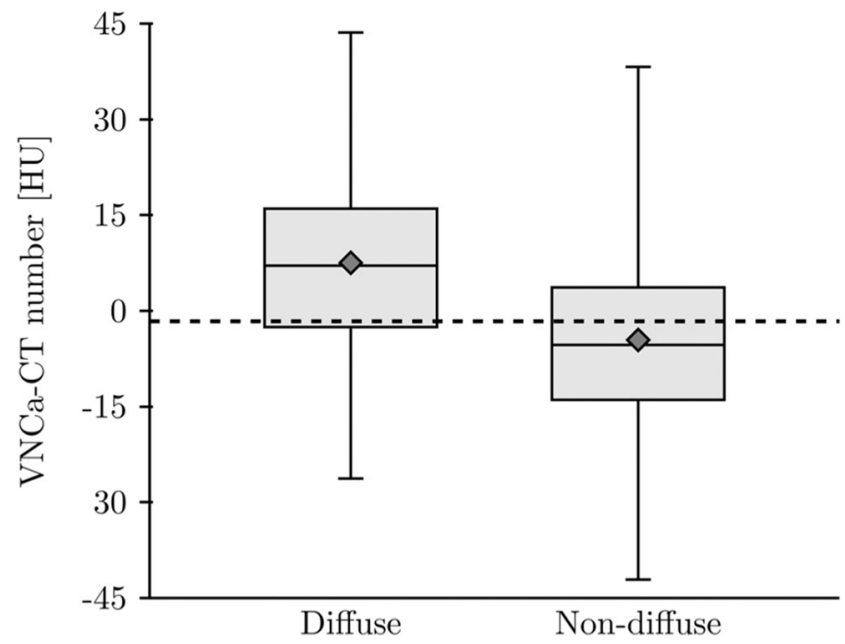

Fig. 5 Boxplots for VNCa-CT numbers for diffuse and non-diffuse infiltration at CaSupp index 65 for mean of L1-5 vertebra. Dashed line: optimum cut-off point of $-1.6 \mathrm{HU}$ for differentiation between both patterns. 
Table 3 Contrast-to-noise ratio for different locations and image data in conventional $\mathrm{CT}$, VNCa$\mathrm{CT}$, and MRI. RIB right iliac bone

\begin{tabular}{lrrrrrrrrr}
\hline & T12 & \multicolumn{1}{l}{ C7 } & \multicolumn{1}{l}{ L1 } & \multicolumn{1}{l}{ L2 } & \multicolumn{1}{l}{ L3 } & L4 & L5 & \multicolumn{1}{l}{ L1- } & RIB \\
& & & & & & & & L5 & \\
\hline Conventional CT & 0.70 & 0.74 & 0.96 & 0.91 & 0.67 & 0.38 & 0.54 & 0.69 & 1.37 \\
CaSupp index 25 & 0.25 & -0.06 & -0.28 & -0.43 & 0.37 & 0.02 & -0.11 & -0.09 & -0.79 \\
CaSupp index 35 & 0.48 & -0.17 & 0.05 & -0.20 & 0.65 & 0.18 & 0.11 & 0.16 & -0.40 \\
CaSupp index 45 & 0.69 & 0.11 & 0.36 & 0.11 & 0.89 & 0.33 & 0.31 & 0.40 & 0.18 \\
CaSupp index 55 & 0.85 & 0.35 & 0.62 & 0.36 & 1.07 & 0.45 & 0.47 & 0.59 & 0.73 \\
CaSupp index 65 & 0.92 & 0.54 & 0.82 & 0.57 & 1.17 & 0.54 & 0.59 & 0.73 & 1.22 \\
CaSupp index 75 & 0.97 & 0.68 & 0.95 & 0.72 & 1.20 & 0.59 & 0.67 & 0.82 & 1.52 \\
CaSupp index 85 & 0.97 & 0.78 & 1.06 & 0.83 & 1.19 & 0.61 & 0.71 & 0.88 & 1.67 \\
CaSupp index 95 & 0.96 & 0.88 & 1.09 & 0.90 & 1.18 & 0.64 & 0.73 & 0.90 & 1.76 \\
MRI ADC & 1.34 & 1.92 & 1.67 & 1.49 & 1.67 & 1.50 & 1.94 & 1.65 & 2.22 \\
\hline
\end{tabular}

For CT number cut-off calculation, we chose to focus on the mean of L1-5 due to the large size of those vertebrae with maximized reliable measurement avoiding sclerosis, fractures, or disc herniations. Although bone marrow involvement in plasma cell dyscrasias is quite heterogeneous, ROImeasurement of the whole lumbar spine in the best performing CaSupp index 65 might be as representative as possible for differentiating diffuse and non-diffuse infiltration, while possible to integrate into clinical routine with acceptable effort.

Using DSCT, Kosmala et al distinguished infiltrated from normal bone marrow with a sensitivity of $93.3 \%$ and a specificity of $92.4 \%$, exceeding the values reported in this study. However, Kosmala et al defined infiltrated marrow independent from the intensity of infiltration and placed ROIs directly on MRI-confirmed focal lesions [14]. In contrast, the main focus of this study is to delineate diffuse infiltration in CT, comprising MRI-confirmed diffuse or intense disseminated focal infiltration. Differences between the DSCT study and this DLCT study are also reflected by differences in cut-off CT numbers (-44.9 HU versus -1.6 HU) [14].

High sensitivities and specificities of $97-100 \%$ were also reached by another recent DSCT study from Kosmala et al differentiating MRI-determined diffuse or focal infiltration patterns from a normal pattern with cut-offs of $35.7 \mathrm{HU}$ and -31.9 HU after placing ROIs in L4-S1, Th12 and the ilium [13]. However, for the differentiation between diffuse and focal pattern, they reported a low sensitivity of $33 \%$ and specificity of $25 \%$. Again, besides different DECT techniques, different approaches of marrow infiltration classification and sites of measurement have to be considered.

Regarding qualitative assessment of $\mathrm{VNCa}-\mathrm{CT}$, a sensitivity and specificity of $91.3 \%$ and $90.9 \%$ was reported for differentiating infiltrated from normal bone marrow using colorcoded DSCT VNCa maps [14], compared to the sensitivity and specificity of $87.8 \%$ and $73.5 \%$ for differentiation between diffuse and non-diffuse infiltration using DLCT VNCa grayscale images in this study, again considering different definitions of infiltration patterns.
In another DSCT study, Thomas et al achieved a sensitivity of $75.0 \%$ and specificity of $82.4 \%$ for the qualitative differentiation of no infiltration versus high-grade infiltration with color-coded maps, but a sensitivity of just $40.0 \%$ and specificity of $85.7 \%$ for the differentiation between no infiltration versus moderate- and high-grade infiltration [18]. For detection of solid tumor metastasis, diagnostic performance can be improved using contrast agent and low CaSupp indices (25 to 50) [19]. However, contrast media are usually not administered to patients with monoclonal plasma cell diseases due to increased risk for renal insufficiency, dose aspects, and the lack of necessity for the detection of osteolysis.

Currently, conventional MRI is the gold standard for bone marrow evaluation, but ADC measurements have been recommended to be added to standard protocols [4, 7]. Our study revealed an ADC cut-off of 487.6 for the differentiation between diffuse and non-diffuse infiltration with a sensitivity of $78.6 \%$ and a specificity of $86.1 \%$. Koutoulidis et al showed higher sensitivity and specificity (100\% and 98\%) for the differentiation of diffuse versus normal bone marrow in MRI [6]. However, aside from different categories of bone marrow involvement, a comparison of specific ADC cut-offs in different studies is still not feasible due to the influence of technical parameters and patient-specific factors [4].

In summary, this study shows an approach for quantitative assessment of bone marrow infiltration in plasma cell dyscrasias in DLCT but further studies have to be performed in order to refine this approach for clinical application. The additional evaluation of spinal bone marrow infiltration in routinely performed CT would be of value, especially for patients with contraindications to MRI. The main limitation of this study is that with the current technical level of visualization in DLCT, it was not feasible to separate the five different infiltration patterns as proposed for MRI [1]. Hence, the main objective was to distinguish widespread infiltration in DLCT from low/no infiltration which was implemented by integration of intense disseminated focal infiltration into the diffuse 
category and sporadic focal infiltration together with the normal pattern into the non-diffuse category. This is in contrast to other studies, which used different definitions of infiltration patterns. Furthermore, future studies should investigate comparability of CT and MRI data acquired by different scanners, imaging protocols, and post-processing tools.

\section{Conclusion}

Quantitative and qualitative assessment of $\mathrm{VNCa}$ images in DLCT is feasible to determine the extent of bone marrow infiltration in plasma cell dyscrasias, especially when the gold standard MRI is not possible. Quantitative assessment of $\mathrm{VNCa}$ images produces comparable results to ADC of MRI at CaSupp index 65, averaging L1-L5 vertebrae, and ROC analysis suggests a cut-off of -1.6 $\mathrm{HU}$ for the differentiation between diffuse and non-diffuse infiltration.

Funding Open Access funding enabled and organized by Projekt DEAL. The authors state that this work has not received any funding.

Dr. Thuy Duong Do was supported by a grant from the Medical Faculty of the University of Heidelberg for research time.

\section{Compliance with ethical standards}

Guarantor The scientific guarantor of this publication is Dr. Thuy Duong Do.

Conflict of interest Dr. Thuy Duong Do was supported by a grant from the Medical Faculty of the University of Heidelberg.

Dr. Stephan Skornitzke has ownership interests in investment funds containing stock of healthcare companies.

Dr. Wolfram Stiller is a member of the CT Advisory Board of Philips Medical Systems.

Otherwise, the authors of this manuscript declare no relationships with any companies, whose products or services may be related to the subject matter of the article or any other conflicts of interest.

Statistics and biometry Dr. Stephan Skornitzke (Applied mathematician) has significant statistical expertise and has a shared first authorship.

Informed consent Written informed consent was waived by the Institutional Review Board.

Ethical approval Institutional Review Board approval was obtained ( $\mathrm{Nr}$. S-348/2019).

Methodology

- retrospective

- randomized controlled trial

- performed at one institution
Open Access This article is licensed under a Creative Commons Attribution 4.0 International License, which permits use, sharing, adaptation, distribution and reproduction in any medium or format, as long as you give appropriate credit to the original author(s) and the source, provide a link to the Creative Commons licence, and indicate if changes were made. The images or other third party material in this article are included in the article's Creative Commons licence, unless indicated otherwise in a credit line to the material. If material is not included in the article's Creative Commons licence and your intended use is not permitted by statutory regulation or exceeds the permitted use, you will need to obtain permission directly from the copyright holder. To view a copy of this licence, visit http://creativecommons.org/licenses/by/4.0/.

\section{References}

1. Baur A, Stabler A, Nagel D et al (2002) Magnetic resonance imaging as a supplement for the clinical staging system of Durie and Salmon? Cancer 95:1334-1345

2. Mai EK, Hielscher T, Kloth JK et al (2015) A magnetic resonance imaging-based prognostic scoring system to predict outcome in transplant-eligible patients with multiple myeloma. Haematologica 100:818-825

3. Mai EK, Hielscher T, Kloth JK et al (2016) Association between magnetic resonance imaging patterns and baseline disease features in multiple myeloma: analyzing surrogates of tumour mass and biology. Eur Radiol 26:3939-3948

4. Messiou C, Hillengass J, Delorme S et al (2019) Guidelines for acquisition, interpretation, and reporting of whole-body MRI in myeloma: Myeloma Response Assessment and Diagnosis System (MY-RADS). Radiology 291:5-13

5. Messiou C, Kaiser M (2015) Whole body diffusion weighted MRI a new view of myeloma. Br J Haematol. https://doi.org/10.1111/ bjh. 13509

6. Koutoulidis V, Fontara S, Terpos E et al (2017) Quantitative diffusion-weighted imaging of the bone marrow: an adjunct tool for the diagnosis of a diffuse MR imaging pattern in patients with multiple myeloma. Radiology 282:484-493

7. Hillengass J, Usmani S, Rajkumar SV et al (2019) International myeloma working group consensus recommendations on imaging in monoclonal plasma cell disorders. Lancet Oncol 20:e302-e312

8. Rajkumar SV, Dimopoulos MA, Palumbo A et al (2014) International Myeloma Working Group updated criteria for the diagnosis of multiple myeloma. Lancet Oncol 15:e538-e548

9. Zamagni E, Nanni C, Patriarca F et al (2007) A prospective comparison of $18 \mathrm{~F}$-fluorodeoxyglucose positron emission tomographycomputed tomography, magnetic resonance imaging and wholebody planar radiographs in the assessment of bone disease in newly diagnosed multiple myeloma. Haematologica 92:50-55

10. Ananthakrishnan L, Duan X, Xi Y et al (2018) Dual-layer spectral detector CT: non-inferiority assessment compared to dual-source dual-energy CT in discriminating uric acid from non-uric acid renal stones ex vivo. Abdom Radiol (NY) 43:3075-3081

11. Sauter AP, Muenzel D, Dangelmaier J et al (2018) Dual-layer spectral computed tomography: virtual non-contrast in comparison to true non-contrast images. Eur J Radiol 104:108-114

12. Schwaiger BJ, Gersing AS, Hammel J et al (2018) Three-material decomposition with dual-layer spectral CT compared to MRI for the detection of bone marrow edema in patients with acute vertebral fractures. Skeletal Radiol 47:1533-1540

13. Kosmala A, Weng AM, Krauss B, Knop S, Bley TA, Petritsch B (2018) Dual-energy CT of the bone marrow in multiple myeloma: 
diagnostic accuracy for quantitative differentiation of infiltration patterns. Eur Radiol 28:5083-5090

14. Kosmala A, Weng AM, Heidemeier A et al (2018) Multiple myeloma and dual-energy CT: diagnostic accuracy of virtual noncalcium technique for detection of bone marrow infiltration of the spine and pelvis. Radiology 286:205-213

15. Landis JR, Koch GG (1977) The measurement of observer agreement for categorical data. Biometrics 33:159-174

16. Di Giuliano F, Picchi E, Muto M et al (2020) Radiological imaging in multiple myeloma: review of the state-of-the-art. Neuroradiology 62:905-923

17. Yu Z, Leng S, Jorgensen SM et al (2016) Evaluation of conventional imaging performance in a research whole-body CT system with a photon-counting detector array. Phys Med Biol 61:15721595
18. Thomas C, Schabel C, Krauss B et al (2015) Dual-energy CT: virtual calcium subtraction for assessment of bone marrow involvement of the spine in multiple myeloma. AJR Am J Roentgenol 204: W324-W331

19. Abdullayev N, Grosse Hokamp N, Lennartz S et al (2019) Improvements of diagnostic accuracy and visualization of vertebral metastasis using multi-level virtual non-calcium reconstructions from dual-layer spectral detector computed tomography. Eur Radiol 29:5941-5949

Publisher's note Springer Nature remains neutral with regard to jurisdictional claims in published maps and institutional affiliations. 